\title{
ATIVIDADES BIOLÓGICAS DO AMIDO RESISTENTE E DE OUTROS OLIGOSSACARÍDEOS NÃO DIGERÍVEIS: UMA BREVE REVISÃO
}

\section{BIOLOGICAL ACTIVITIES OF RESISTANT STARCH AND OTHER NONDIGESTIBLE OLIGOSACCHARIDES: A BRIEF REVIEW}

\author{
Marjana Radünz'1, Bruna Luiza Paulina Ribas², Gabrielle Gaspar Arejano², Taiane \\ Mota Camargo', Helen Cristina dos Santos Hackbart', André Luiz Radünz ${ }^{3}$
}

1 - Programa de Pós-Graduação em Ciência e Tecnologia de Alimentos - Universidade Federal de Pelotas, RS

2 - Programa de Pós-Graduação em Nutrição e Alimentos - Universidade Federal de Pelotas, RS

3 - Docente do Departamento de Agronomia - Universidade Federal da Fronteira Sul - Campus Chapecó, SC Autor correspondente: marjanaradunz@gmail.com

\section{RESUMO}

Atualmente a população tem buscado uma alimentação mais saudável devido ao aumento das doenças crônicas não transmissíveis, entre os alimentos com atividades biológicas protetores se encontram 0 amido resistente e outros oligossacarídeos. $O$ objetivo do presente trabalho foi realizar uma breve revisão sistemática utilizando bases de dados indexadas acerca dos benefícios do amido resistente e de outros oligossacarídeos não digeríveis sobre a saúde humana. Como resultado da breve revisão evidenciou-se que os oligossacarídeos não-digeríveis e o amido resistente apresentam diversos benefícios para a saúde, atuando principalmente sobre o funcionamento do trato gastrointestinal, sistema nervoso central, sistema imunológico, biodisponibilidade de nutrientes, prevenção do câncer colorretal e na redução dos níveis bioquímicos de glicose, triglicerídeos e colesterol. Palavras chave: disponibilidade biológica, trato gastrointestinal, alimento funcional

\section{ABSTRACT}

Currently the population has sought a healthier diet due to the increase of chronic noncommunicable diseases, among foods with protective biological activities are resistant starch and other oligosaccharides. The objective of the present work was to conduct a brief systematic review using indexed databases on the benefits of resistant starch and other non-digestible oligosaccharides on human health. Because of the brief review it was evidenced that non-digestible oligosaccharides and resistant starch present several health benefits, acting mainly on gastrointestinal tract functioning, central nervous system, immune system, nutrient bioavailability, colorectal cancer prevention and in reducing the biochemical levels of glucose, triglycerides and cholesterol.

Keywords: biological availability, gastrointestinal tract, functional food

\section{INTRODUÇÃO}

Nos últimos anos tem havido um aumento substancial de interesse no estudo de alimentos funcionais, que possuem potenciais benefícios para a saúde. Segundo o Ministério da Saúde alimento funcional é todo aquele alimento ou ingrediente que, além das 
funções nutricionais básicas, quando consumido na dieta usual, produz efeitos metabólicos e/ou fisiológicos benéficos à saúde, devendo ser seguro para o consumo, sem supervisão médica (BRASIL, 1999a; BRASIL, 1993b). Dentre este grupo, encontram-se os oligossacarídeos não digeríveis (OND) definidos como, compostos prebióticos que apresentam características físico-químicas importantes e propriedades fisiológicas benéficas para a saúde. Os OND são considerados carboidratos e sua estrutura consiste em unidades de glicose, nas quais são em média, 2 a 10 monômeros de frutosil, galactosil entre outros monômeros que estão ligados por ligações glicosídicas que não podem ser digeridos ou absorvidos pelo estômago, no entanto, entram num processo de fermentação no intestino delgado que estimula o crescimento de Bifidobacterium spp. e Lactobacillus spp., organismos benéficos à saúde do cólon (PEREIRA, 2007; MEHRA \& KELLY, 2006; VAN LOO et al., 2005).

Os galacto-oligossacarídeos (GOS) são formados pela transgalactosilação da galactose a partir de substratos ricos em lactose através da ação da enzima $\beta$-galactosidase (MAHONEY, 1998). Podem ser encontrados na soja e sintetizados a partir da lactose (VAN LOO et al., 2005). Os fruto-oligossacarídeos (FOS) são oligossacarídeos naturais, que são encontrados principalmente em produtos de origem vegetal. Estão presentes em grande parte na inulina, e estima-se que seu consumo nesta forma oscila entre 1 a $4 \mathrm{~g} / \mathrm{dia}$ (VAN LOO et al., 2005).

O amido resistente, é definido como a quantidade total de amido e produtos derivados da degradação de amidos resistentes à digestão no intestino delgado de pessoas saudáveis, considerando então, o amido resistente como uma fibra dietética total. Esse amido é encontrado em grãos, batata crua, banana verde, ou mesmo em alimentos processados e retrogradados como a casca de pão ou a batata cozida resfriada e o consumo atual diário é de aproximadamente 3g/pessoa (PEREIRA, 2007). Pode ser utilizado em substituição ao amido digestível da alimentação, pois ele reduz a glicemia e a insulina, além disto, ele reduz calorias de alimentos e aumenta a oxidação de lipídeos reduzindo a gordura corporal (JIANG et al., 2010). Diferentemente de fibra que é definida de acordo com a resolução RDC 40/2001da ANVISA/MS como qualquer material comestível que não seja hidrolisado pelas enzimas endógenas do trato digestivo humano, determinado segundo os métodos publicados pela AOAC em sua edição mais atual. No entanto, apresentam efeitos benéficos bastante semelhantes, tais como a resistência a hidrólise enzimática no trato gastrointestinal diminuindo a absorção de glicose e aumenta a fermentação microbiana de ácidos de cadeia curta no intestino (CHUNG et al., 2011). 
Diante do crescente interesse da utilização dos alimentos funcionais, fazem-se necessárias maiores pesquisas para elucidar os benefícios e questionamentos encontrados a cerca de seus subgrupos. Os oligossacarídeos não digeríveis, principalmente o amido resistente, tem sido alvo de grandes pesquisas pela crescente preocupação com a saúde da população. O objetivo do presente trabalho é realizar uma revisão sistemática a respeito dos benefícios dos oligossacarídeos não digeríveis e do amido resistente à saúde.

\section{METODOLOGIA}

Este trabalho abordou o tema sobre o papel do amido resistente e de outros oligossacarídeos não digeríveis sobre todos os aspectos benéficos para a saúde da população. A metodologia empregada foi o estudo exploratório-descritivo através de pesquisa bibliográfica e da utilização de dados secundários oriundos de publicações e resultados de pesquisas específicas sobre 0 assunto. Foram utilizadas as bases de dados Science Direct, Scielo, PubMed e Lilacs com buscas de artigos e livros utilizando os seguintes descritores: oligossacarídeos não digeríveis, amido resistente, oligossacarídeos não digeríveis e saúde, amido resistente e saúde, non-digestible oligosaccharides and health e resistant starch and health. Selecionou-se os artigos que apresentavam as características relevantes ao objetivo da revisão nos últimos 20 anos.

\section{EFEITOS BIOLÓGICOS DOS OLIGOSSACARÍDEOS}

\section{1 Galacto-oligossacarídeos}

Os galacto-oligossacarídeos (GOS) apresentam um maior efeito antiaderente na parede do trato gastrointestinal, melhorando o trânsito intestinal, o metabolismo de minerais, carboidratos e lipídios; um menor residual de lactose reduzindo o risco de intolerância; um menor residual de açucares metabolizáveis, reduzindo cáries e teor calórico. Ainda, pode ser uma alternativa para alimentação de pacientes críticos e com riscos de infecção (MARTINS \& BURKET, 2009). Varasteh et al (2015), realizou estudo afim de avaliar se o GOS pode proteger a barreira intestinal de células com adenocarcinoma colorretal submetidas ao estresse térmico, concluiu que este impediu a suprarregulação induzida pelo estresse em proteínas de choque térmico e por consequência, houve redução da resposta ao estresse induzido pelo calor. O GOS apresenta ainda, um importante 
potencial na prevenção e na progressão do câncer colorretal segundo um estudo de revisão de Bruno-Barcena et al (2015).

\subsection{Oligossacarídeos não digeríveis e sistema nervoso central}

Em estudo realizado na Alemanha, Lehmann et al (2015) propuseram-se a avaliar os efeitos imunomoduladores de FOS e GOS sobre células dendríticas e observaram que a mistura destes oligossacarídeos apresentou atividade anti-inflamatória e ação imunomoduladora sobre estas.

\subsection{Oligossacarídeos não digeríveis e trato gastrointestinal}

Bielecka et al (2002), observaram que a suplementação de FOS e lactulose apresentou efeito laxativo em ratos devido a intensificação da fermentação intestinal.

\subsection{Oligossacarídeos não digeríveis e parâmetros bioquímicos}

Os oligossacarídeos não digeríveis regulam os níveis de glicose, pois não são quebrados com facilidade, deste modo o açúcar proveniente da sua digestão demora mais tempo para ficar disponível para as células, sendo descarregado na circulação aos poucos fazendo com que a insulina não seja requisitada a todo instante. Pesquisas também demostram que os oligossacarídeos atuam na diminuição do colesterol LDL e dos níveis de triglicerídeos (PEREIRA, 2007). Van Dokkun et al (1999), realizou um estudo para avaliar o efeito da ingestão de OND em parâmetros de função intestinal sobre as concentrações de lipídios no sangue e absorção de glicose e concluiu que estes são fermentados em parte no cólon, mas não encontrou alterações nos parâmetros bioquímicos avaliados.

\subsection{Oligossacarídeos não digeríveis e sistema imunológico}

Em um estudo realizado por Van Viles et al (2012), demonstrou que a suplementação dietética de FOS e GOS em ratas grávidas não melhorou o retardo das respostas de hipersensibilidade, mas tem um efeito diferencial em alguns marcadores imunológicos tanto na mãe como no feto. Em contrapartida, as ratas não grávidas do grupo controle apresentaram um aumento do retardo destas respostas quando suplementadas. 


\subsection{Oligossacarídeos não digeríveis e biodisponibilidade de nutrientes}

Os oligossacarídeos não digeríveis são associados com aumento na biodisponibilidade de certos minerais como ferro, cálcio, magnésio e zinco. A maior disponibilidade de zinco favorece a síntese proteica e a conversão da vitamina A para sua forma ativa. Atua também aumentando a acidez nos cólons, devido o aumento da fermentação microbiana, essa variação de acidez aumenta a solubilidade dos minerais no ceco e nos cólons, e conduz a maior absorção dos minerais (PEREIRA, 2007). Um estudo avaliando a suplementação de FOS em ratos observou uma diminuição da excreção fecal de minerais, indicando uma melhoria da absorção de cálcio, magnésio, ferro e zinco e uma diminuição da excreção urinaria de nitrogênio, sugerindo um deslocamento de uma parte da excreção deste para o intestino grosso.

\subsection{Amido resistente e indústria}

Em 2015 no Brasil, Silva et al avaliou os potenciais usos da farinha de banana verde (FBV) obtidos por diferentes métodos para elaboração de produtos alimentícios. A FBV promove o aumento do teor de amido resistente, compostos fenólicos e atividade antioxidante e também atua na redução do índice glicêmico dos produtos tornando-se uma alternativa para a composição da dieta de indivíduos diabéticos e obesos. Vernaza et al (2011) observou ainda, que a adição de 10\% de FBV na fabricação de macarrão instantâneo reduziu significativamente o teor de gordura em todos os tempos de fritura e aumento o teor de amido resistente quando comparado ao macarrão instantâneo comercial.

\subsection{Amido resistente e saúde}

Em um estudo realizado por Koh et al (2016) com intuito de analisar se a alimentação com amido resistente pode modular as concentrações circulantes de adiponectina e angiotensina II, bem como a adiposidade e os alvos da angiotensina II no rim, que tem sido associada a nefropatia diabética e avaliar se o equilíbrio de vitamina $D$ em ratos com diabetes mellitus tipo 2. Concluiu-se que o amido resistente não teve impacto sobre as concentrações de glicose no sangue e percentual de hemoglobina glicada. Adiponectina circulante foi $77 \%$ maior em ratos alimentados com amido resistente comparado aos ratos alimentados com amido de milho. Evidenciou-se também que o 
equilíbrio de vitamina $D$ na presença de hiperglicemia é fortemente associada com níveis de adiponectina e redução do sinal do sistema renina-angiotensina.

\subsection{Amido resistente e biodisponibilidade de nutrientes}

Bosscher et al (2003), observou que a adição de fibra dietética solúvel afeta o cálcio, ferro, zinco e disponibilidade de inulina positiva ou negativamente dependo do tipo de fibra dietética utilizada.

\section{CONCLUSÃO}

Conclui-se que os OND e o amido resistente apresentam potenciais benéficos para a saúde, principalmente sobre o trato gastrointestinal auxiliando no transito e na absorção dos nutrientes.

\section{REFERÊNCIAS}

BIELECKA, M.; BIEDRZYCKA, E.M.B.; MAJKOWSKA, A.; JUSKIEWICZ, J.J.; WROBLEWSKA, M. Effect of non-digestible oligosaccharides on gut microecosystem in rats. Food Research International, v.35, p.139-144, 2002.

BOSSCHER, D.; CAILLIE-BERTRAND, M.V.; CAUWENBERGH, R.V.; DEELSTRA, H. Availabilities of calcium, iron, and zinc from dairy infant formulas is affected by soluble dietary fibers and modified starch fractions. Nutrition, v.19, n. 7-7, p. 641-645, 2003.

BRASIL, AGÊNCIA NACIONAL DE VIGILÂNCIA SANITÁRIA. ANVISA. Resolução $\mathrm{n}$ ㄴ 18, de 30 de abril de 1999. Aprova o Regulamento Técnico que Estabelece as Diretrizes Básicas para Análise e Comprovação de Propriedades Funcionais e ou de Saúde Alegadas em Rotulagem de Alimentos. Diário Oficial da República Federativa do Brasil. Brasília,03 nov. 1999.

BRASIL, AGÊNCIA NACIONAL DE VIGILÂNCIA SANITÁRIA. ANVISA. Resolução ㄲo 19, de 30 de abril de 1999. Aprova o Regulamento Técnico de Procedimentos para Registro de Alimento com Alegação de Propriedades Funcionais e ou de Saúde em sua Rotulagem.

Diário Oficial da República Federativa do Brasil. Brasília, 10 dez. 1999. 
BRASIL. Resolução RDC n.40, de 21 de marco de 2001. A Diretoria Colegiada da ANVISA/MS aprova o regulamento técnico para rotulagem nutricional obrigatória de alimentos e bebidas embalados. Diário Oficial da União. 200122 mar; (57-E): 22.

BRUNO-BARCENA, J.M.; AZCARETE, M.A. Galacto-oligosaccharides and colorectal cancer: Feeding our intestinal probiome. Journal of Functional Foods, v.16, p. 92-108, 2015.

CHUNG, H.J.; DONNER, E.; LIU, Q. Resistant Starch in Foods. In: MOO-YOUNG, M. Comprehensive Biotechnology: agriculture and related biotechnologies. 2. ed. Amsterdam: Elvesier, 2011. Capítulo 4, p. 527-534.

JIANG, H.; HORNER, H.T.; PEPPER, T.N.; BLANCO, N.; CAMPBELL, M.; JANE, J. Formation of elongated starch granules in high-amylose maize. Carbohydrate Polymers, v.80, n.2., p. 533-538, 2010.

KOH, G.Y.; DERSCHEID, R.; FULLER, K.N.Z.; VALENTINE, R.J.; LEOW, S.E.; REED, L.; WISECUP, E.; SCHALINSKE, K.L.; ROWLING, M.J. Circulating adiponectin concentrations are increased by dietary resistant starch and correlate with serum 25-hydroxycholecalciferol concentrations and kidney function in Zucker diabetic fatty rats. Nutrition research, v.36, p. $311-319,2016$.

LEHMANN, S.; HILLER, J.; VAN BERGENHENEGOUWEN, J.; KNIPPELS, L.M.J.;, GARSSEN, J.; TRAIDL-HOFFMANN, C. In Vitro Evidence for Immune-Modulatory Properties of Non-Digestible Oligosaccharides: Direct Effect on Human Monocyte Derived Dendritic Cells. Plos One, v.10, n.7, 2015.

MAHONEY, R.R. Galactosyl-oligosaccharide formation during lactose hydrolysis: a review. Food Chemistry, v.63, n.2, p.147-154, 1998.

MARTINS, A.R.; BURKERT, C.A.V. Galacto-oligossacarídeos (GOS) e seus efeitos prebióticos e bifidogênicos. Brazilian Journal of Food Technology, v.12, n.3, p. 230-240, 2009.

MEHRA, R.; KELLY, P. Milk oligosaccharides: structural and technological aspects. International Dairy Journal, v. 16, n. 11, p. 1334-1340, 2006. 
PEREIRA, K.D. Amido resistente, a última geração no controle de energia e digestão saudável. Ciência e Tecnologia de Alimentos, v.27, p. 88-92, 2007.

SILVA, A.A.; JUNIOR, J.L.B.; BARBOSA, M.I.M.J. Farinha de banana verde como ingrediente funcional em produtos alimentícios. Ciência Rural, v.45, n.12, 2015.

VAN DOKKUM, W.; WEZENDONK, B.; SRIKUMAR, T.S.; VAN DEN HEUVEL, E.G.H.M. Effect of nondigestible oligosaccharides on large-bowel functions, blood lipid concentrations and glucose absorption in young healthy male subjects. European Journal of Clinical Nutrition, v.53, p. 1-7, 1999.

VAN LOO, J.; COUSSEMENT, P.; DE LEENHEER, L.; HOEBERGS, H.; SMITS, G. On the presence of inulin and oligofructose as natural ingredients in the Western diet. Reviews in Food Science and Nutrition, v.35, n.6, p. 525-552, 1995.

VAN VILES, N.; HOGENKAMPA, A.; THIJSSENA, S.; DINGJANA, G.M.; KNIPPINGA, K.; GARSSENA, J.; KNIPPELS, L.M.J. Effects of short-chain galacto- and long-chain fructooligosaccharides on systemic and local immune status during pregnancy. Journal of Reproductive Immunology, v.94, p.161-168, 2012.

VARASTEH, S.; BRABER, S.; GARSSEN, J.; FINK-GREMMELS, J. Galactooligosaccharides exert a protective effect against heat stress in a Caco-2 cell model. Journal of Functional Foods, v.16, p. 265-277, 2015.

VERNAZA, M.G.; GULARTE, M.A.; CHANG, Y.K. Adição de farinha de banana verde em macarrão instantâneo: propriedades reológicas e tecnológicas. Ciência e Agrotecnologia, v.35, n.6, p. 1157-1165, 2011. 